\title{
Secuencias Educativas: el docente digitalizado y la percepción de la enseñanza
}

\section{Educational Sequences: digitized teacher and the perceptions of teaching}

\author{
Eduardo Dopico Rodríguez \\ Universidad de Oviedo \\ dopicoeduardo@uniovi.es
}

\section{Resumen}

El alumnado universitario que se prepara para el ejercicio docente se encuentra, mediante las prácticas externas, con la oportunidad de contrastar en la realidad práctica los conocimientos teóricos y procedimentales que adquieren en las aulas universitarias. Al otro lado del pupitre, de pie en el aula, se encuentran por primera vez frente a niños y adolescentes sentados que les miran y esperan de su capacidad para trasladarles conocimientos empleando múltiples fuentes de información.

Tomando como referencia esta situación de las prácticas externas, se presentan los resultados de una investigación basada en la percepción que sobre la competencia profesional docente tienen 120 estudiantes, futuros maestros y profesores, en el ámbito de la educación primaria y secundaria en centros educativos asturianos a lo largo de 3 cursos académicos. Interesa conocer las relaciones causales y considerar críticamente como ven y experimentan las prácticas docentes reales, como piensan que es educarse hoy en la era digital de qué forma valoran el rol del profesorado en activo, el modelo curricular, el método didáctico, las posibilidades efectivas para la innovación educativa $y$, finalmente, su propia competencia para afrontar los procesos de enseñanza-aprendizaje con un grupo-clase.

Palabras-clave: didáctica, competencia, prácticas externas, enseñanza digitalizada 


\begin{abstract}
University students preparing for the teaching practice is through external training, with the opportunity to compare the practical reality and procedural knowledge acquired in university classrooms. Across the desk, standing in the classroom, meet for the first time in front of children and teenagers sitting watching and waiting them of their ability to pass on knowledge using multiple sources of information.
\end{abstract}

Drawing on this situation of internships, we present the results of an investigation based on the perception on teacher professional competition have 120 students, future teachers, in the field of primary and secondary education in Asturian schools to over 3 academic years. Interest to know the causal relationships and consider critically as they see and experience real teaching practices, as they think it is to educate today's digital age how teachers value the active role, the model curriculum, the teaching method, the actual possibilities for educational innovation and, ultimately, its own competence to meet the teaching-learning process with a-class.

Key words: teaching, competition, internships, teaching digitized

Fecha recepción: Agosto 2012 Fecha aceptación: Diciembre 2012

\title{
Introducción
}

En el ámbito europeo de educación, el proceso de convergencia educativa, denominado Proceso de Bolonia (http://es.wikipedia.org/wiki/Proceso de Bolonia), anima a cambiar los viejos esquemas de enseñanza para adaptar las metodologías docentes tanto a las necesidades actuales de los estudiantes universitarios como a la competitividad global del mercado de trabajo. Quizá el aspecto más visible de este cambio resida en el progresivo abandono de la enseñanza tradicional, orientada al aprendizaje de contenidos, para estimular el desarrollo de procesos educativos 
basados en el aprendizaje de competencias, tratando de dar sentido a lo que se aprende (Martínez y Escarbajal, 2011). Si bien este cambio abarca a todas las titulaciones universitarias, es en el ámbito de la pedagogía donde tiene mayor garantía de penetración, de trascendencia social. Las Ciencias de la Educación siempre se han interesado en revelar las características de hecho educativo. Abordar la enseñanza del proceso didáctico, el cómo se aprende a enseñar, es una preocupación latente de la investigación educativa. Desde este punto de vista, la formación del profesorado se convierte en objetivo prioritario del cambio conceptual y metodológico que la sociedad digital actual requiere. Así, desde la Universidad, es grato comprobar la cantidad de jóvenes, futuros profesionales de la educación (Vaillant y Manso, 2012) que se matriculan en los títulos universitarios tomando la pedagogía y el magisterio como primera opción. Si a ellos les sumamos al alumnado de otras disciplinas que se acercan al perfil docente a través de la acreditación específica, nos encontramos con una bolsa de recursos personales fiable y competente que garantiza el relevo generacional en las aulas y nuevas perspectivas que producirán ganancias de aprendizaje y enfoques didácticos innovadores de los que nos beneficiaremos todos. Tenemos el material humano. Nuestro compromiso es ofrecerles las mejores oportunidades de aprendizaje posibles (Puelles, 2009; Marcelo y Yot, 2011).

Durante su proceso de aprendizaje, quienes estudian para convertirse en profesores pasan por diferentes etapas. Una que siempre concita estrés e ilusión son las prácticas externas en centros educativos. La duración de las prácticas varía de unos títulos de Grado a otros y se sitúan en un intervalo que va de los 44 ECTS del Grado de Primaria a los 13 ECTS del título de Master en Formación del Profesorado (ECTS: European Credit Transder System; el Sistema Europeo de Transferencia de Créditos es una medida del trabajo de entre 25 a 30 horas, que realiza el estudiante para cumplir con los objetivos del programa de estudios). Las prácticas externas facilitan los primeros contactos con la práctica docente, con la realidad cotidiana de la escuela, con ese momento en que el/la estudiante asume el papel de profesor/a (Marcelo, 2009). Permiten analizar y valorar el funcionamiento del colegio y la dinámica del aula desarrollando el hábito de la observación constructiva y el análisis sistemático (Dopico y Garcia-Vázquez, 2010). Representan el marco donde participar de la actividad docente contrastando los conocimientos que ya poseen con los que se aplican en el 
espacio del grupo-clase (Valle y Manso, 2011). En este contexto, el alumnado universitario que se prepara para el ejercicio docente se encuentra por primera vez al otro lado del pupitre, de pie en el aula, frente a niños y adolescentes sentados que les miran y esperan de su capacidad para trasladarles conocimientos empleando múltiples fuentes de información. Los profesores que participamos en su formación tratamos de optimizar la formación práctica de los estudiantes a partir del estudio de la transferencia de competencias profesionales en centros de prácticas y nos preguntamos acerca del paisaje educativo que se encontrarán en los centros, sobre cómo percibirán esa mutación de estudiante a docente en prácticas y que aprendizajes extraerán de todo esto para su futuro desarrollo profesional.

En ocasiones, coincide que algún alumno/a docente en prácticas acude a realizar las prácticas externas al mismo centro donde cursó sus estudios primarios o secundarios. Esta vez, con sus recuerdos de alumno/a, llevan consigo los conocimientos que reciben en las Facultades de Educación: conocimientos teóricos precisos sobre la práctica docente, criterios rigurosos de observación e intervención educativa y pautas claras de actuación didáctica (Novella et al, 2012). Al ser nativos digitales (Prensky, 2001), les acompaña su considerable competencia digital en entornos 2.0, sus experiencias con los lenguajes audiovisuales y las redes virtuales del espacio 3.0 y sus capacidades en el tratamiento y transferencia de la información (Snyder, (2004). A todo esto suman sus ideas previas acerca del rol docente (Barberà, 2008), para acomodar la práctica a la imagen que tienen de sí mismos y a los estereotipos del profesor que quieren imitar o rehuir. A pesar de los esfuerzos por aclarar que ser docente no es sencillo y requiere una formación sólida, existe la creencia de que enseñar es fácil, que prepararse para enseñar consiste en aprender cómo hacer las cosas y que se aprende de la experiencia (Woolfolk y Murphy, 2001). El feed back (realimentación) que recibimos cuando regresan de esas estancias externas en los centros de prácticas, nos ayuda a reflexionar acerca de las sutilezas y las carencias que estructuran nuestros sistemas educativos. A construir conocimiento y reformular lo que se ha aprendido y cobrado significado en compañía de otros (Rosaen y Florio Ruane-2008), para seguir avanzando hacia los conocimientos que aún no se poseen. El balance conjunto entre el profesorado que formamos a los futuros docentes y los propios estudiantes, permite reducir la distancia entre lo que ya conocen y lo que 
deberían conocer, entre cómo conocen y cómo lo deberían conocer cuando la escuela ya cuente efectivamente con ellos (Ruiz y Martínez, 2010).

\section{MATERIAL Y MÉTODO}

Tomando como referencia las Prácticas Externas, se plantea una estrategia de investigación basada en el estudio de casos (Stake, 2005), para acercarnos a la percepción que sobre la competencia profesional docente tiene el alumnado-docente en prácticas, en el ámbito de la Educación Primaria (dirigida a estudiantes de 6 a 12 años) y Secundaria (dirigida a estudiantes de entre 12 y 16 años). Siguiendo su experiencia individual concreta, se toma como marco de referencia para el análisis, las estancias externas (el Prácticum) de estudiantes del título de Grado en Primaria (68 alumnos/as) y del Master en Formación del Profesorado (52 alumnos/as) en centros educativos asturianos a lo largo de 3 cursos académicos. De los 120 estudiantes tomados como muestra representativa, 27 eran alumnos y 93 alumnas (Figura 1).

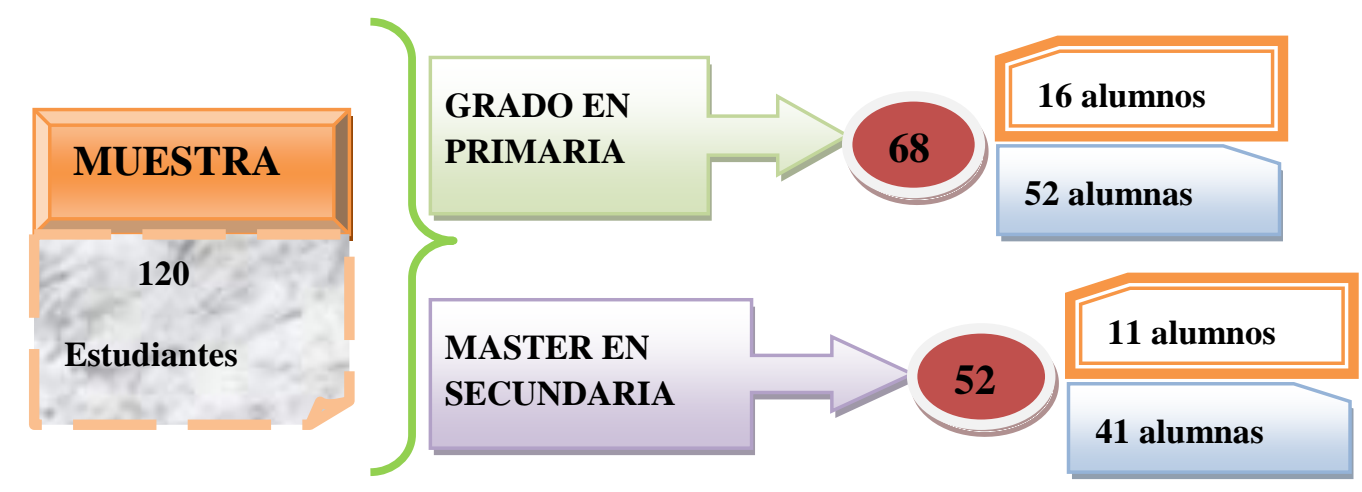

Figura 1: Muestra analizada

Interesa conocer las relaciones causales y considerar críticamente como ven y experimentan unos y otros las prácticas docentes reales, como piensan que es educarse hoy en la era digital (Pérez Gómez, 2012), de qué forma valoran el rol del profesorado en activo, el modelo curricular, el método didáctico, las posibilidades efectivas para la innovación educativa $y$, finalmente, su propia competencia para afrontar los procesos de enseñanza-aprendizaje con un grupo-clase. Para poder analizar datos fiables y válidos desde un enfoque cualitativo y cuantitativo que permita un análisis riguroso de la información proporcionada por la muestra generadora de datos, se elabora un Cuestionario de preguntas sustantivas a la investigación que, a 
través de ítems dicotómicos y categorizados (Hernández et al, 2000; Martínez Olmo, 2002), permite recoger la información relevante de manera sistemática y ordenada (Tabla 1). Este cuestionario fue pasado de modo presencial y anónimo a ambos grupos de estudiantes después de cada una de sus fases de prácticas a lo largo de 3 años académicos (2009-2012).

\begin{tabular}{|c|c|}
\hline PERCEPCIÓN & _ Qué característica/s piensas que definen el rol docente \\
\hline & $\begin{array}{l}\text { Señala los modelos curriculares que te parecen más adecuados en la } \\
\text { enseñanza }\end{array}$ \\
\hline DOCENTE & _ Cuáles son los métodos educativos con los que más te identificas \\
\hline $\begin{array}{l}\text { EN } \\
\text { PRÁCTICAS }\end{array}$ & $\begin{array}{l}\text { _ Elige una sola opción: el profesorado elige el método más adecuado } \\
\text { para conseguir los objetivos didácticos }\end{array}$ \\
\hline 2009-2012 & $\begin{array}{l}\text { Elige una sola opción: en los Centros escolares no se puede hacer } \\
\text { innovación porque lo impide... }\end{array}$ \\
\hline $\begin{array}{l}\text { ETAPA } \\
\text { EDUCATIVA }\end{array}$ & $\begin{array}{l}\text { Elige las opciones que mejor definan si durante las prácticas en el } \\
\text { Centro pudiste comprobar... }\end{array}$ \\
\hline
\end{tabular}

Tabla 1: Cuestionario

Para determinar la fiabilidad de los datos que proporciona el Cuestionario y las tendencias en la relación entre ítems, se sigue el coeficiente de consistencia interna de Cronbach, midiendo la respuesta de cada elemento de la muestra con respecto a los ítems del instrumento para, posteriormente, a través del coeficiente de correlación de Pearson, medir la magnitud de la relación entre dichos ítems. En ambas pruebas, coeficientes de fiabilidad con valores que oscilan sobre 0,9 dan credibilidad al instrumento diseñado (Gil Pascual, 2006; Visauta, 2007). Para el tratamiento estadístico de los datos se dicotomiza la muestra sobre su población - diana (futuros/as profesores/as de Primaria y de Secundaria, respectivamente), sometiendo cada Cuestionario a un análisis descriptivo con ayuda del paquete SPSS 19 (Pérez, 2005; Rodríguez et al. 2007)

\section{RESULTADOS}


Como se decía antes, los Cuestionarios son pasados una vez que los alumnos y alumnas regresan a las aulas, después de su estancia en los centros educativos de prácticas. Recogida esta información, los datos tabulados presentan estos resultados (Tablas 2 y 3 ).

\begin{tabular}{|c|c|c|c|c|c|c|}
\hline \multicolumn{7}{|c|}{$\begin{array}{l}\text { PERCEPCIÓN DEL ALUMNADO DOCENTE EN } \\
2012 \\
\text { GRADO EN PRIMARIA }\end{array}$} \\
\hline & \multicolumn{2}{|c|}{ Alumnos } & Alumnas & \begin{tabular}{l|l} 
S & Tota \\
\end{tabular} & & \\
\hline Alumnado & \multicolumn{2}{|c|}{$16(23,5 \%)$} & $\begin{array}{l}52 \\
(76,5 \%)\end{array}$ & 68 & & \\
\hline \multicolumn{7}{|c|}{$\begin{array}{l}\text { Qué característica/s piensas que definen el rol } \\
\text { docente }\end{array}$} \\
\hline & $\begin{array}{l}\text { Profesio } \\
\text { nal }\end{array}$ & \multicolumn{2}{|c|}{$\begin{array}{l}\text { Comprensiv } \\
\text { o }\end{array}$} & $\begin{array}{l}\text { Competen } \\
\text { te }\end{array}$ & $\begin{array}{l}\text { Motivad } \\
\text { or }\end{array}$ & $\begin{array}{l}\text { Comunicati } \\
\text { vo }\end{array}$ \\
\hline Alumnos & $6(37,5 \%)$ & \multicolumn{2}{|c|}{$2(12,5 \%)$} & $12(75 \%)$ & $5(31 \%)$ & $4(25 \%)$ \\
\hline Alumnas & $10(19 \%)$ & \multicolumn{2}{|c|}{$15(29 \%)$} & 31 (60\%) & $16(31 \%)$ & $11(21 \%)$ \\
\hline Total & $\begin{array}{l}16 \\
(23,5 \%)\end{array}$ & \multicolumn{2}{|c|}{$17(25 \%)$} & $30(44 \%)$ & 21 (31\%) & $15(22 \%)$ \\
\hline
\end{tabular}

\begin{tabular}{|c|c|c|c|}
\hline \multicolumn{4}{|l|}{$\begin{array}{l}\text { Señala los m } \\
\text { enseñanza }\end{array}$} \\
\hline & Alumnos & Alumnas & Total \\
\hline $\begin{array}{l}\text { El Modelo tecnológico (por } \\
\text { objetivos) }\end{array}$ & $8(50 \%)$ & $12(23 \%)$ & $\begin{array}{l}20 \\
(29 \%)\end{array}$ \\
\hline $\begin{array}{l}\text { El Modelo de investigación en la } \\
\text { acción }\end{array}$ & $5(31 \%)$ & $16(31 \%)$ & $\begin{array}{l}21 \\
(31 \%)\end{array}$ \\
\hline $\begin{array}{l}\text { Los Modelos situacionales } \\
\text { (artísticos) }\end{array}$ & $5(31 \%)$ & $\begin{array}{l}19 \\
(36,5 \%)\end{array}$ & $\begin{array}{l}24 \\
(35 \%)\end{array}$ \\
\hline Los Modelos Socio-críticos & $\begin{array}{l}10 \\
(62,5 \%)\end{array}$ & $35(67 \%)$ & $\begin{array}{l}45 \\
(66 \%)\end{array}$ \\
\hline
\end{tabular}




\begin{tabular}{|c|c|c|c|}
\hline \multicolumn{4}{|c|}{$\begin{array}{l}\text { Cuáles son los métodos educativos con los que más te } \\
\text { identificas }\end{array}$} \\
\hline & Alumnos & Alumnas & Total \\
\hline El método Expositivo / Lección Magistral & $8(50 \%)$ & $2(3,8 \%)$ & $\begin{array}{l}10 \\
(15 \%)\end{array}$ \\
\hline El método de Simulación y Juego & $12(75 \%)$ & $48(92 \%)$ & $\begin{array}{l}60 \\
(88 \%)\end{array}$ \\
\hline El método de Estudio de Casos & \begin{tabular}{|l|}
2 \\
$(12,5 \%)$
\end{tabular} & $\begin{array}{l}19 \\
(36,5 \%)\end{array}$ & $\begin{array}{l}21 \\
(31 \%)\end{array}$ \\
\hline El método del Contrato de Aprendizaje & $1(6,25 \%)$ & $16(31 \%)$ & $\begin{array}{l}17 \\
(25 \%)\end{array}$ \\
\hline El método del Aprendizaje Cooperativo & $13(81 \%)$ & $34(65 \%)$ & $\begin{array}{l}47 \\
\text { (69\%) }\end{array}$ \\
\hline $\begin{array}{l}\text { El método del Aprendizaje Basado en } \\
\text { Problemas (ABP) }\end{array}$ & $11(69 \%)$ & $36(69 \%)$ & $\begin{array}{l}47 \\
(69 \%)\end{array}$ \\
\hline
\end{tabular}

Elige una sola opción: el profesorado elige el método más adecuado para conseguir los objetivos didácticos

\begin{tabular}{|c|c|c|c|c|}
\hline & Sí & No & \multicolumn{2}{|c|}{$\begin{array}{l}\text { Tiene que seguir el método } \\
\text { que ya se está } \\
\text { desarrollando en el Centro }\end{array}$} \\
\hline $\begin{array}{l}\text { Alumn } \\
\text { os }\end{array}$ & $2(12,5 \%)$ & $1(6 \%)$ & \multicolumn{2}{|l|}{$13(81 \%)$} \\
\hline $\begin{array}{l}\text { Alumn } \\
\text { as }\end{array}$ & $26(50 \%)$ & $5(10 \%)$ & \multicolumn{2}{|l|}{$21(40 \%)$} \\
\hline Total & $28(41 \%)$ & $6(9 \%)$ & \multicolumn{2}{|l|}{$34(50 \%)$} \\
\hline \multicolumn{5}{|c|}{$\begin{array}{l}\text { Elige una sola opción: en los Centros escolares no se pu } \\
\text { hacer innovación porque lo impide }\end{array}$} \\
\hline & \multicolumn{2}{|c|}{$\begin{array}{l}\text { El tiempo que } \\
\text { tienes para dar tu } \\
\text { asignatura }\end{array}$} & $\begin{array}{l}\text { La dinámica de } \\
\text { trabajo creada ya } \\
\text { en el centro }\end{array}$ & \begin{tabular}{|l} 
Se \\
puede
\end{tabular} \\
\hline Alumn & \multicolumn{2}{|l|}{$9(56 \%)$} & $6(37,5 \%)$ & $1(6 \%)$ \\
\hline
\end{tabular}




\begin{tabular}{|c|c|c|c|c|c|}
\hline \multicolumn{6}{|l|}{ OS } \\
\hline $\begin{array}{l}\text { Alumn } \\
\text { as }\end{array}$ & $9(17 \%)$ & $9(17 \%)$ & $\begin{array}{l}34 \\
(65 \%)\end{array}$ & & \\
\hline Total & $18(26 \%)$ & $15(22 \%)$ & $\begin{array}{l}35 \\
(51 \%)\end{array}$ & & \\
\hline \multicolumn{6}{|c|}{$\begin{array}{l}\text { Elige las opciones que mejor definan si durante las prácticas en el Centro pudiste } \\
\text { comprobar }\end{array}$} \\
\hline & & & Alumnos & Alumna & Total \\
\hline \multicolumn{3}{|c|}{$\begin{array}{l}\text { Que puedo afrontar el reto de llevar una clase y } \\
\text { seguir una programación }\end{array}$} & \begin{tabular}{l|l}
16 \\
$(100 \%)$
\end{tabular} & $\begin{array}{l}38 \\
(73 \%)\end{array}$ & $\begin{array}{l}54 \\
(79 \%)\end{array}$ \\
\hline \multicolumn{3}{|c|}{$\begin{array}{l}\text { Que aún me queda mucho para llegar a ser el / la } \\
\text { profesor/a que quiero ser }\end{array}$} & $5(31 \%)$ & $\begin{array}{l}44 \\
(85 \%)\end{array}$ & $\begin{array}{l}49 \\
(72 \%)\end{array}$ \\
\hline \multicolumn{3}{|c|}{$\begin{array}{l}\text { Que siguen enseñando con las mismas técnicas que } \\
\text { emplearon cuando yo estaba en esta etapa } \\
\text { educativa }\end{array}$} & 15 (94\%) & $\begin{array}{l}49 \\
(92 \%)\end{array}$ & $\begin{array}{l}64 \\
(94 \%)\end{array}$ \\
\hline
\end{tabular}

Tabla 2: Resultados netos del Cuestionario del Grado Primaria

\begin{tabular}{|c|c|c|c|c|c|c|}
\hline \multicolumn{7}{|c|}{$\begin{array}{l}\text { PERCEPCIÓN DEL ALUMNADO DOCENTE EN PRÁCTICAS 2009-2012 } \\
\text { MASTER EN SECUNDARIA }\end{array}$} \\
\hline & Alumnos & Alumnas & $\begin{array}{l}\text { Tota } \\
\text { I }\end{array}$ & ta & & \\
\hline $\begin{array}{l}\text { Alumnad } \\
\text { o }\end{array}$ & $11(21 \%)$ & 41 (79\%) & \multicolumn{2}{|c|}{52} & & \\
\hline \multicolumn{7}{|c|}{$\begin{array}{l}\text { Qué característica/s piensas que definen el rol } \\
\text { docente }\end{array}$} \\
\hline & $\begin{array}{l}\text { Profesiona } \\
\text { I }\end{array}$ & \multicolumn{2}{|c|}{$\begin{array}{l}\text { Comprensiv } \\
\text { o }\end{array}$} & $\begin{array}{l}\text { Competen } \\
\text { te }\end{array}$ & $\begin{array}{l}\text { Motivad } \\
\text { or }\end{array}$ & $\begin{array}{l}\text { Comunicati } \\
\text { vo }\end{array}$ \\
\hline Alumnos & $8(73 \%)$ & $5(45 \%)$ & & $9(82 \%)$ & 5 (45\%) & $6(54,5 \%)$ \\
\hline Alumnas & $13(32 \%)$ & $10(24 \%)$ & & 33 (80\%) & $18(44 \%)$ & $11(27 \%)$ \\
\hline Total & $21(40 \%)$ & $15(29 \%)$ & & $27(52 \%)$ & $23(44 \%)$ & $17(33 \%)$ \\
\hline
\end{tabular}




\begin{tabular}{|c|c|c|c|c|}
\hline $\begin{array}{l}\text { Señala los modelos curriculares q } \\
\text { enseñanza }\end{array}$ & ue te parec & cen más a & decuados & \\
\hline & \begin{tabular}{|l|l} 
Alumno & $A$ \\
$\mathrm{~s}$ & $\mathrm{~s}$
\end{tabular} & $\begin{array}{l}\text { Alumna } \\
\mathrm{s}\end{array}$ & Total & \\
\hline $\begin{array}{l}\text { El Modelo tecnológico (por } \\
\text { objetivos) }\end{array}$ & 9 (81\%) & $\begin{array}{l}11 \\
(27 \%)\end{array}$ & $\begin{array}{l}20 \\
(38 \%)\end{array}$ & \\
\hline $\begin{array}{l}\text { El Modelo de investigación en la } \\
\text { acción }\end{array}$ & $2(18 \%)$ & $\begin{array}{l}19 \\
(46 \%)\end{array}$ & $\begin{array}{l}21 \\
(40 \%)\end{array}$ & \\
\hline $\begin{array}{l}\text { Los Modelos situacionales } \\
\text { (artísticos) }\end{array}$ & $1(9 \%)$ & $\begin{array}{l}17 \\
(41 \%)\end{array}$ & $\begin{array}{l}18 \\
(35 \%)\end{array}$ & \\
\hline Los Modelos Socio-críticos & $4(36 \%)$ & $\begin{array}{l}29 \\
(71 \%)\end{array}$ & $\begin{array}{l}33 \\
(63 \%)\end{array}$ & \\
\hline $\begin{array}{l}\text { Cuáles son los métodos educativc } \\
\text { identificas }\end{array}$ & os con los q & que más $t$ & & \\
\hline & & $\begin{array}{l}\text { Alumn } \\
\mathrm{s}\end{array}$ & \begin{tabular}{l|l} 
& Alumna \\
$\mathrm{s}$
\end{tabular} & Total \\
\hline El método Expositivo / Lección Mag & gistral & $\begin{array}{l}10 \\
(91 \%)\end{array}$ & $6(15 \%)$ & $\begin{array}{l}16 \\
(31 \%)\end{array}$ \\
\hline El método de Simulación y Juego & & $2(18 \%$ & $\begin{array}{l}14 \\
(34 \%)\end{array}$ & $\begin{array}{l}16 \\
(31 \%)\end{array}$ \\
\hline El método de Estudio de Casos & & $\begin{array}{l}2 \\
(18 \%)\end{array}$ & $\begin{array}{l}18 \\
(44 \%)\end{array}$ & $\begin{array}{l}20 \\
(38 \%)\end{array}$ \\
\hline El método del Contrato de Aprend & lizaje & $1(9 \%)$ & $\begin{array}{l}16 \\
(39 \%)\end{array}$ & $\begin{array}{l}17 \\
(33 \%)\end{array}$ \\
\hline El método del Aprendizaje Cooper & ativo & $3(27 \%$ & $\begin{array}{l}31 \\
(76 \%)\end{array}$ & $\begin{array}{l}34 \\
(65 \%)\end{array}$ \\
\hline $\begin{array}{l}\text { El método del Aprendizaje } \\
\text { Problemas (ABP) }\end{array}$ & Basado en & \begin{tabular}{l|l}
$127 \%$ \\
\end{tabular} & $\begin{array}{l}36 \\
(88 \%)\end{array}$ & $\begin{array}{l}39 \\
(75 \%)\end{array}$ \\
\hline
\end{tabular}

Elige una sola opción: el profesorado elige el método más adecuado para conseguir los objetivos didácticos

\begin{tabular}{|l|l|l|}
\hline Sí & No & Tiene que seguir el método \\
\hline
\end{tabular}




\begin{tabular}{|c|c|c|c|c|c|c|}
\hline & & & $\begin{array}{l}\text { que ya se } \\
\text { desarrollando en el } \mathrm{C} \epsilon\end{array}$ & $\begin{array}{l}\text { está } \\
\text { entro }\end{array}$ & & \\
\hline Alumnos & $2(18 \%)$ & 0 & $9(82 \%)$ & & & \\
\hline Alumnas & $\begin{array}{l}23 \\
(57 \%)\end{array}$ & $4(10 \%)$ & $14(34 \%)$ & & & \\
\hline Total & 9 (17\%) & 5 (10\%) & $38(73 \%)$ & & & \\
\hline \multicolumn{5}{|c|}{$\begin{array}{l}\text { Elige una sola opción: en los Centros escolares no se puede } \\
\text { hacer innovación porque lo impide }\end{array}$} & \\
\hline & \multicolumn{2}{|c|}{$\begin{array}{l}\text { El tiempo que } \\
\text { tienes para dar tu } \\
\text { asignatura }\end{array}$} & $\begin{array}{l}\text { La dinámica de } \\
\text { trabajo creada ya } \\
\text { en el centro }\end{array}$ & $\begin{array}{l}\text { Se } \\
\text { puede }\end{array}$ & & \\
\hline Alumnos & \multicolumn{2}{|l|}{$4(36 \%)$} & $7(63,6 \%)$ & 0 & & \\
\hline Alumnas & \multicolumn{2}{|l|}{$11(27 \%)$} & $10(24 \%)$ & $\begin{array}{l}20 \\
(49 \%)\end{array}$ & & \\
\hline Total & \multicolumn{2}{|l|}{$15(29 \%)$} & $17(33 \%)$ & $\begin{array}{l}20 \\
(77 \%)\end{array}$ & & \\
\hline \multicolumn{7}{|c|}{$\begin{array}{l}\text { Elige las opciones que mejor definan si durante las prácticas en el Centro pudiste } \\
\text { comprobar }\end{array}$} \\
\hline & & & & Alumnos & Alumnas & Total \\
\hline \multicolumn{4}{|c|}{$\begin{array}{l}\text { Que puedo afrontar el reto de llevar una clase y } \\
\text { seguir una programación }\end{array}$} & $\begin{array}{l}11 \\
(100 \%)\end{array}$ & 35 (85\%) & $\begin{array}{l}46 \\
(88 \%)\end{array}$ \\
\hline \multicolumn{4}{|c|}{$\begin{array}{l}\text { Que aún me queda mucho para llegar a ser el / la } \\
\text { profesor/a que quiero ser }\end{array}$} & $3(45 \%)$ & 34 (83\%) & $\begin{array}{l}39 \\
(75 \%)\end{array}$ \\
\hline \multicolumn{4}{|c|}{$\begin{array}{l}\text { Que siguen enseñando con las mismas técnicas que } \\
\text { emplearon cuando yo estaba en esta etapa } \\
\text { educativa }\end{array}$} & $\begin{array}{l}11 \\
(100 \%)\end{array}$ & $\begin{array}{l}40 \\
(97,5 \%)\end{array}$ & $\begin{array}{l}51 \\
(98 \%)\end{array}$ \\
\hline
\end{tabular}

Tabla 3: Resultados netos del Cuestionario del Master Secundaria

Veamos estos datos que arrojan los 120 Cuestionarios de forma más precisa:

$\checkmark$ Características del rol docente (Figura 2)

El 71\% los/as 120 estudiantes que responden al Cuestionario, piensa que el rasgo que mejor define a un/a profesor es el hecho de que sea "Competente". Si nos fijamos en 
las otras características señaladas por los estudiantes, se destaca el valor" Profesional" escogido por algo más de la mitad de los estudiantes y el perfil de "Motivador" elegido por el $36,5 \%$ de las alumnas

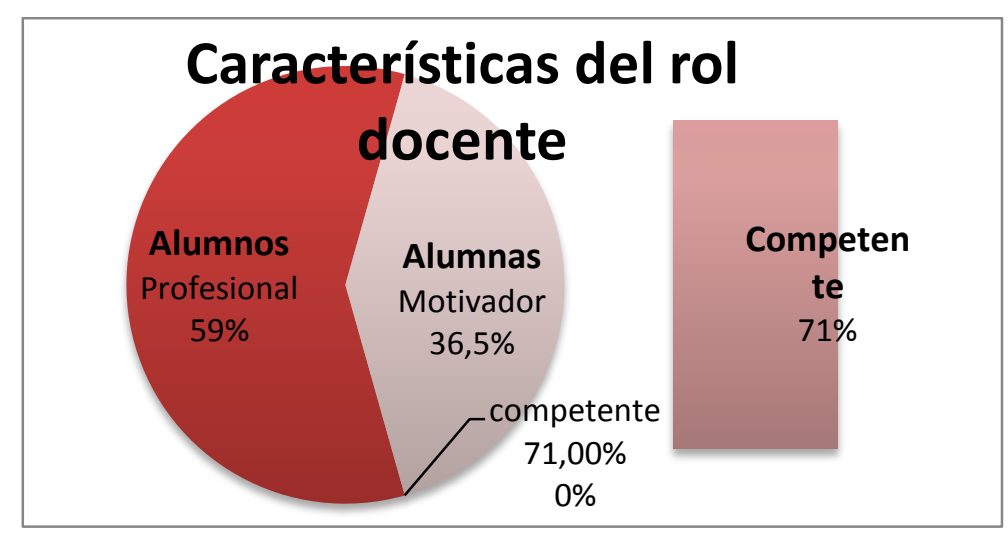

Figura 2: Características del rol docente

\section{$\checkmark$ Modelos curriculares preferidos (Figura 3)}

Entre los diferentes modelos curriculares que se estudian en la Facultad de Formación del Profesorado y Educación, el alumnado seguido durante sus prácticas docentes a lo largo de estos 3 años académicos muestra un sesgo de género evidente al decantarse por el modelo curricular de su preferencia. Si para los alumnos, el clásico modelo orientado a objetivos, el modelo Tecnológico es el modelo con más adeptos (63\%), las alumnas abren el campo curricular eligiendo los más actuales modelos sociocríticos $(69 \%)$

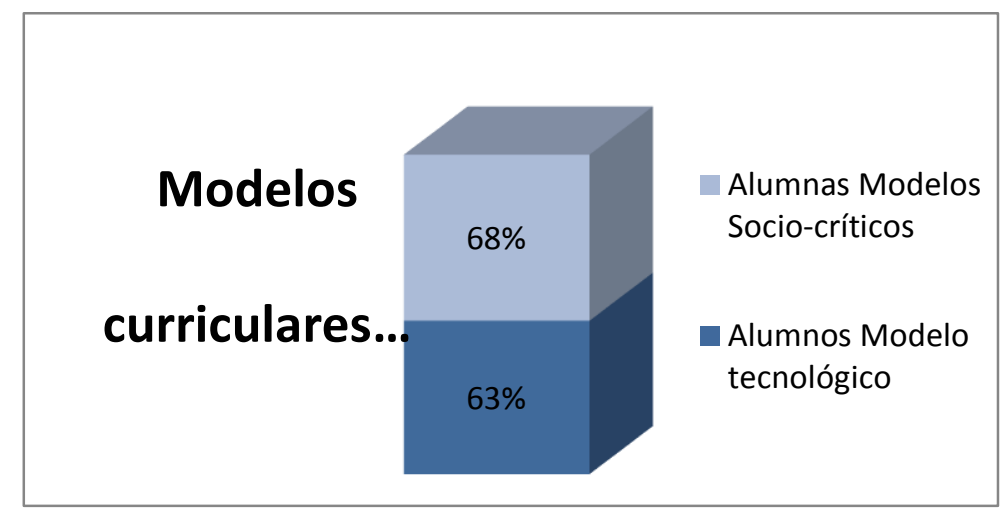

Figura 3: Modelos curriculares elegidos

\section{$\checkmark$ Métodos educativos con los que te identificas}


Los futuros docentes de la etapa de Educación Primaria se decantan por el método de Simulación y Juego (75\% y 92 \% respectivamente), cuando piensa en el enfoque didáctico que más les gusta. Si introducimos la variable género en la elección metodológica, observamos cómo el $81 \%$ de los estudiantes también se muestran partidarios de utilizar como referente de la práctica didáctica el Aprendizaje Cooperativo (81\%). Las alumnas de esta especialidad docente además, eligen el Aprendizaje Basado en Problemas (69\%) como estrategia de enseñanza (Figura 4).

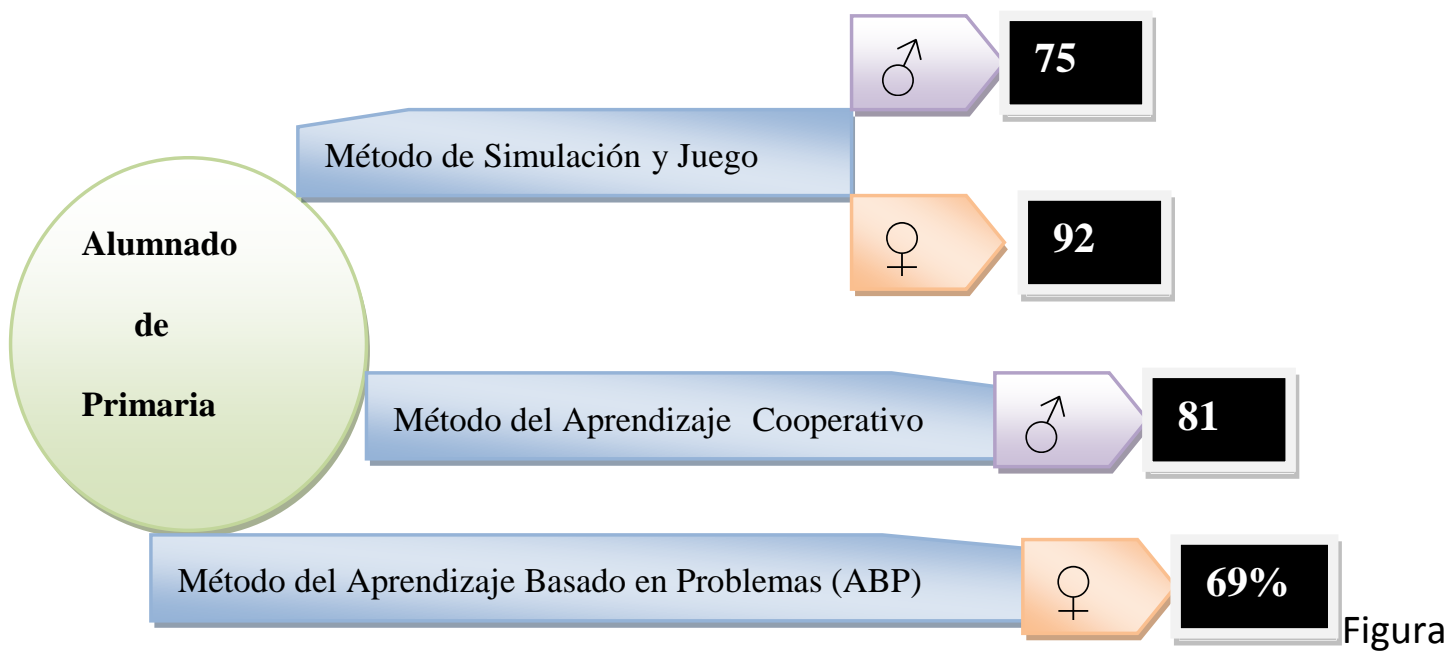

4: Modelos educativos preferidos por los fututos docentes de Primaria

Por su parte, los estudiantes del Master en Formación del Profesorado revelan una percepción algo antigua de la función docente. Se ve claramente en su elección metodológica: casi en su totalidad (91\%), se decanta por el método discursivo de la Lección Magistral. No ocurre así entre las estudiantes. Éstas, abren las posibilidades a otras formas de ejercer la docencia. Así, consideran el método del Aprendizaje Cooperativo (76\%) y el método del Aprendizaje Basado en Problemas (88\%), como metodologías de enseñanza más afines a su manera de entender la docencia (Figura 5).

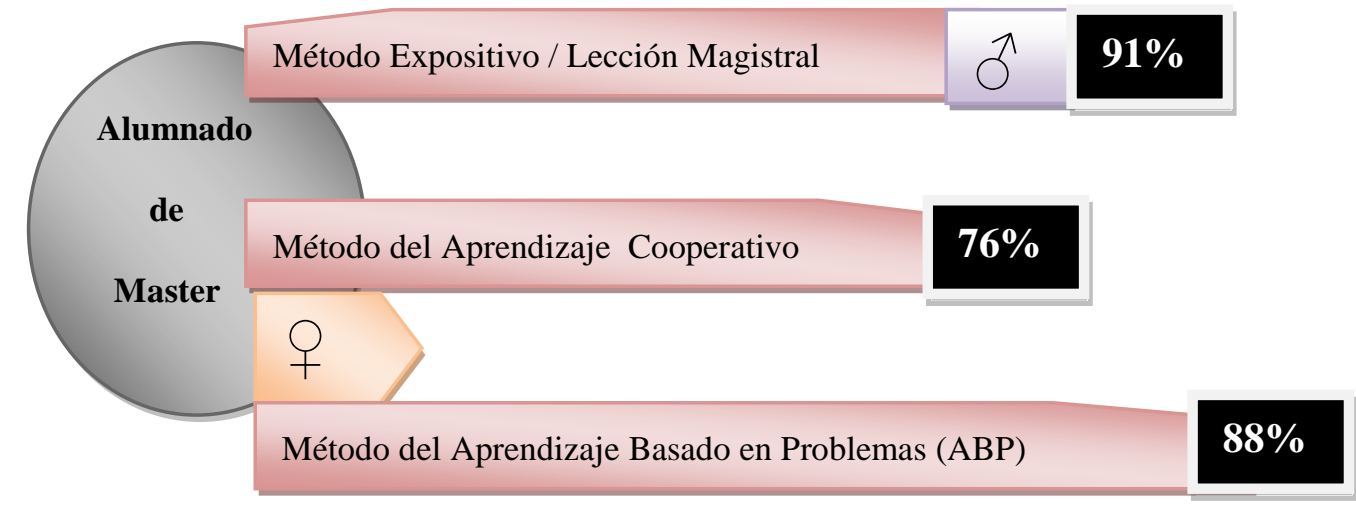


Figura 5: Modelos educativos preferidos por los fututos docentes de Secundaria

$\checkmark$ ¿El profesorado elige el método más adecuado para conseguir los objetivos que pretenda alcanzar con sus alumnas/os? (Figura 6)

Una cosa es que los/as alumnos/as tengan ideas respecto a cómo ejercitar la enseñanza y otra, la realidad que observan a través de las prácticas. Aquí se les ponía en la disyuntiva de aclarar si el docente elige el método didáctico o tiene que reproducir el estandarizado en el centro educativo. Tanto en Primaria como en el Master (78\%), los alumnos parece que tienen claro que deben adaptarse al método que ya se desarrolla en el Centro Educativo. Sin embargo, las alumnas de una y otra etapa confieren al docente mayor autonomía y capacidad de actuación metodológica (53\%)

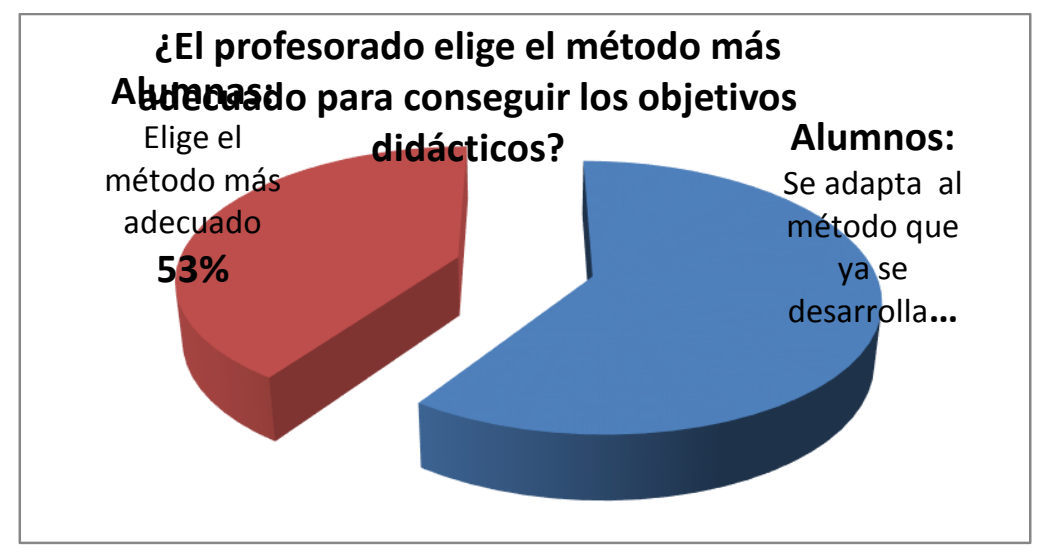

Figura 6: Elección del método didáctico

V En los Centros escolares no se puede hacer innovación porque lo impide....

Cuando se plantea la posibilidad o no de la innovación educativa (Figura 7), observamos de nuevo un sesgo de género: el $45 \%$ de las alumnas creen que es posible innovar. Sorprende ver que entre los alumnos ni siquiera es considerada la posibilidad de innovar en las aulas. Solo 1 alumno de Primaria eligió esa opción. Los alumnos no lo tienen nada claro. De hecho el $27,5 \%$ de ellos cree que lo impide el tiempo que se tiene para impartir la asignatura y el $27 \%$ piensa que lo impide la dinámica de trabajo creada ya en el centro 

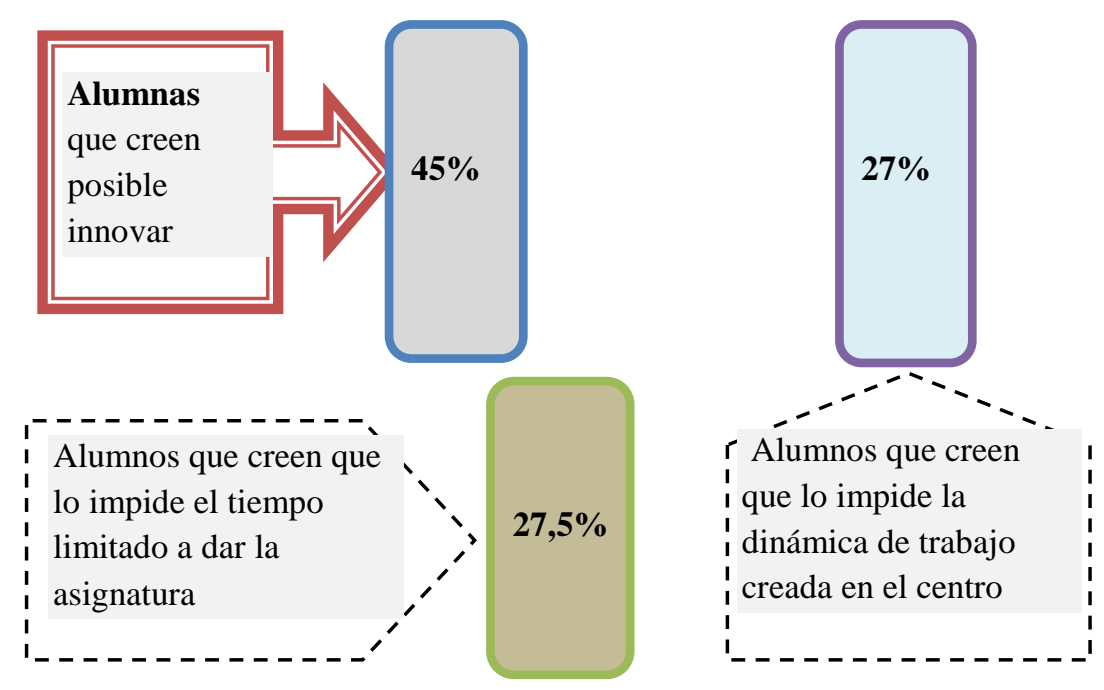

Figura 7: Posibilidades de innovación

$\checkmark$ Elige las opciones que mejor defina si durante las prácticas en el Centro pudiste comprobar....

Los alumnos de un nivel y otro muestran seguridad en sus capacidades para situarse al frente del grupo-aula: el $100 \%$ de los alumnos y el $78 \%$ de las alumnas, está convencido de su competencia para ello (Figura 8).

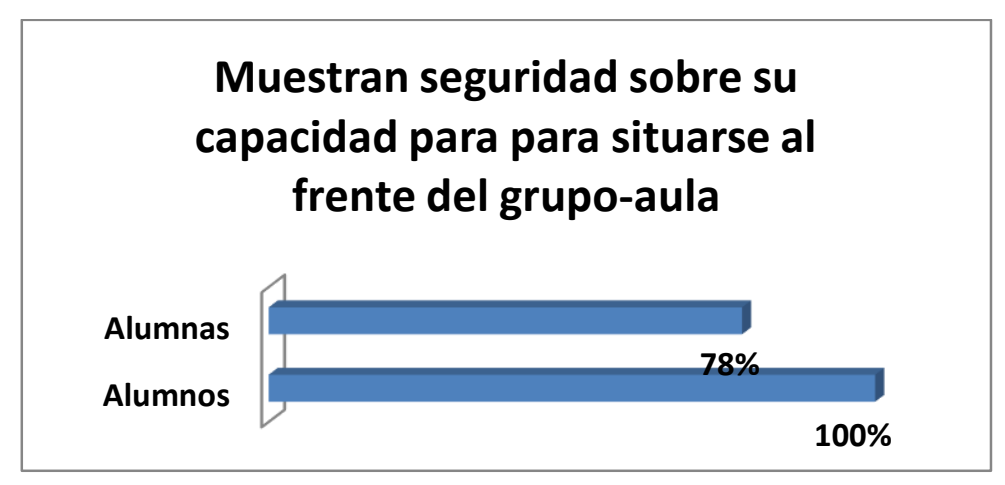

Figura 8: Percepción de la propia capacidad docente

Como tratamos con alumnos en formación, cuestionarles sobre cómo valoran la propia competencia adquirida nos ofrece una imagen nítida del efecto que las prácticas externas les provocan. Así, el $70 \%$ de los alumnos señala rotundo que ya es el profesor que quiere ser. Las alumnas, nuevamente, revelan un criterio más sensato: el $83 \%$ aún piensa en que necesita mejorar su competencia como futura docente (Figura 9). 


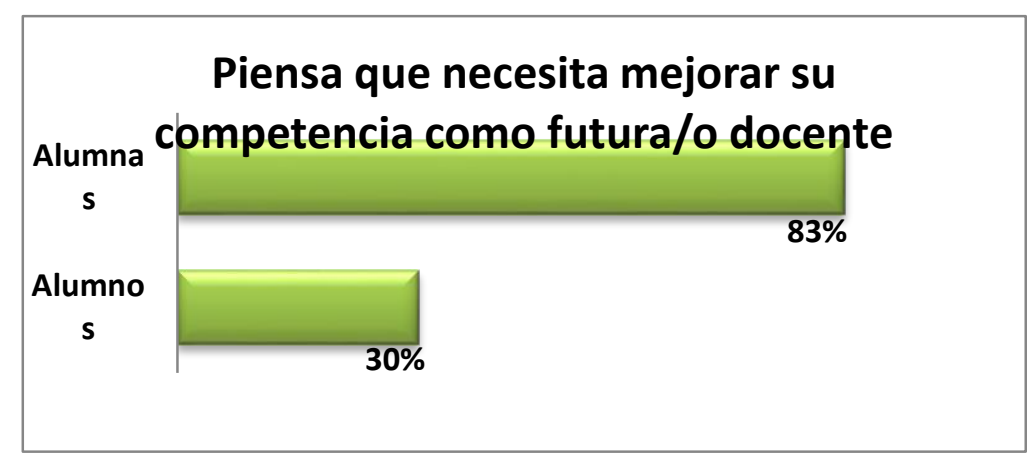

Figura 9: Percepción sobre las necesidades de mejora

Donde no cabe duda alguna es en la percepción que unos y otras tienen del inmovilismo en la práctica pedagógica. El 96\% del alumnado seguido durante su periodo de prácticas externas regresa a las aulas universitarias asegurando que en la enseñanza primaria y secundaria se sigue enseñando con las mismas técnicas que empleaban sus antiguos profesores cuando ellas y ellos cursaban estas etapas (Figura 10)

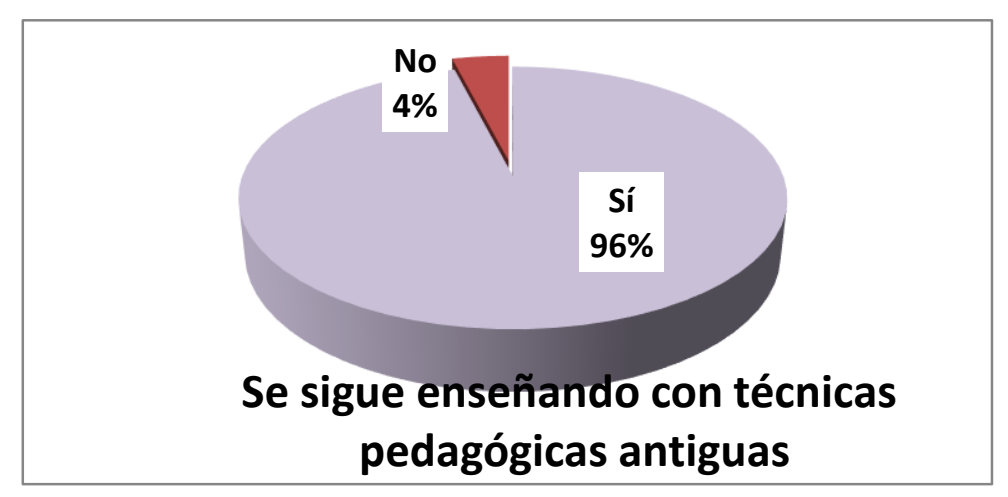

Figura 10: Valoración de los métodos de enseñanmza observados 


\section{Conclusiones}

Con las prácticas externas, los estudiantes, futuros docentes, tienen la oportunidad de contrastar en la realidad práctica los conocimientos teóricos y procedimentales que adquieren en las aulas universitarias. Como necesitan poner en valor las competencias que van obteniendo, pueden visualizar su significatividad y los grados de dominio que ya poseen al ejercitarlas ante un grupo-clase. La experiencia formativa que suponen las horas pasadas como docentes en prácticas en los centros educativos les acerca a entender, desde dentro, cómo funciona el proceso de enseñanza-aprendizaje, cómo se construye el andamiaje instruccional (Bruner, 1996) y cómo se desenvuelve el campo profesional del profesorado (Latorre y Blanco, 2011). Será una vez que regresan a la Facultad cuando comienzan a tomar verdadera conciencia de lo experimentado y a otorgar valor a la formación previa recibida. Aunque no todos. Algunos de entre ellos, cuando vuelven de las prácticas externas, sobre todo los que cursan el master en secundaria, manifiestan poco aprecio por los conocimientos previos ofrecidos en los programas formativos y otorgan el mérito exclusivo del programa docente a la oportunidad de vivir la experiencia del Prácticum (Shkedi y Laron, 2004).

Se observa el interés de los y las estudiantes encuestados en caracterizar como competente la imagen que tienen del mejor profesor posible. Esa competencia profesional refleja un perfil docente más ajustado a la realidad humana del grupo-clase y al momento vital que vivimos. Un docente competente es quien sabe adaptarse a las necesidades y ritmos de aprendizaje de sus alumnos/as, a las condiciones del contexto y del programa educativo. Aquel que es capaz de reflexionar sobre la comunicación didáctica que establece. Así lo entiende el $71 \%$ de la muestra analizada. Sin embargo, al dicotomizar a los estudiantes entre alumnos y alumnas vemos cómo el $63 \%$ de los alumnos diluye esa idea del profesor competente al preferir un modelo curricular como el Modelo Tecnológico, basado en objetivos y representado por la enseñanza discursiva y la lección magistral. Afortunadamente las alumnas hacen elecciones más consecuentes con las opiniones expresadas y el $69 \%$ de ellas manifiesta preferir los modelos sociocríticos para desarrollar los procesos de enseñanza-aprendizaje, 
reconociendo la necesidad de poner en práctica enfoques metodológicos diferentes que absorban y codifiquen los conocimientos más actualizados posibles para estimular y apoyar con la máxima eficacia el aprendizaje de sus alumnos (Day, 2006).

Cuando el Cuestionario plantea un ítem en interrogación, se pretende que el sujeto muestral concrete su opinión entre opciones predeterminadas. En este caso, sobre la capacidad del profesorado para elegir la metodología más adecuada a la práctica didáctica. Como se veía antes, el aprendizaje basado en competencias enlaza con los requerimientos a poner el reloj docente al día, actualizando la metodología y la organización de la docencia (Murillo, 2007). Los alumnos, tanto en la especialidad docente en Primaria como en Secundaria aún revelan una mentalidad gregaria cuando el $78 \%$ de ellos dice que hay que adaptarse al método que ya se desarrolla en el Centro Educativo. Las alumnas están más convencidas de la autonomía de criterio y la capacidad de actuación metodológica de los docentes.

Otra de las modalidades elegidas para la elaboración de ítems en el Cuestionario fue la formulación negativa de una de las cuestiones. En este apartado, para tratar el aspecto de la innovación. Sabemos que en estos casos la puntuación más alta esperada suele corresponder al mayor desacuerdo con la opinión expresada en el ítem (Morales Vallejo, 2011) y así ocurre entre las alumnas, donde el $45 \%$ de ellas ve factible la innovación. Pero aquí volvemos a apreciar un sesgo de género. El acuerdo con la imposibilidad de innovar en los centros educativos es prácticamente total entre los alumnos: tan solo un estudiante del Grado de Educación Primaria pensaba que innovar era viable. Cuando las dificultades para establecer algún tipo de innovación de corte metodológico en las aulas se basa en el tiempo limitado que se tiene para impartir la asignatura o se argumenta que lo impide la dinámica de trabajo creada ya en el centro, se adopta una posición acomodaticia que reproduce los males educativos que se critican. En muchas ocasiones, innovar tiene que ver con trabajar, con dedicar tiempo previo a preparar las clases, los contenidos, las actividades, con introducir en las dinámicas educativas nuevas herramientas, planteamientos distintos.

Dedicar 44 créditos ECTS o 13 a realizar prácticas externas en los centros educativos también ayuda a los docentes en prácticas ajustar la imagen que tienen de sí mismos como docentes. Los contenidos profesionalizantes de las materias que cursan en las Facultades de Educación les confieren seguridad en sí mismos. Así lo 
revelan los datos analizados cuando vemos como el $100 \%$ de los alumnos y el $78 \%$ de las alumnas manifiestan sentirse capacitados para asumir el papel de docente. Por supuesto, asumir el rol no significa ser docente, y esto lo tienen más claro las alumnas que los alumnos. Mientras la mayoría de ellas piensa que aún necesita continuar formándose para llegar a ajustarse a la imagen que tienen del docente ideal, el exceso de autoconfianza entre el $70 \%$ de los alumnos cuando aseguran ser ya el profesor que quieren ser, resulta fatuo.

Cuando se los pregunta a los y las estudiantes de la muestra por los métodos educativos con los que se identifican, se está intentando validar nuevamente la cuestión sobre los modelos curriculares preferidos. Pero al hablar del método, la influencia de la formación recibida se revela como un factor a tener en cuenta, al menos entre los alumnos. Los estudiantes del Grado de Primaria piensan que las metodologías participativas que requieren dinámicas permanentes en el aula, tales como el método de Simulación y Juego o el método del Aprendizaje Cooperativo describen mejor su personalidad como futuros docentes. Estos estudiantes aún cursan el título de Grado. No así los alumnos del Master de Secundaria, que ya son todos licenciados universitarios. Éstos eligen mayoritariamente el método discursivo de la Lección Magistral como referente de su actuación futura como docentes. Se podría establecer algún sesgo motivado por el nivel de estudios, pero esta circunstancia no se da entre las alumnas de una y otra especialidad. No cabe tampoco notoriedad diferencial alguna de género como vemos. Tal vez la explicación más aproximada a la certeza sea fijar el hecho a las características individuales de las personas que responden a la cuestión.

El Cuestionario finaliza con una afirmación tan rotunda por parte de quienes lo cumplimentan acerca del inmovilismo didáctico, que preocupa. El 96\% del alumnado que participó en esta investigación (115 estudiantes de 120), asegura haber comprobado, en persona, que en la enseñanza primaria y secundaria se sigue enseñando con las mismas técnicas que empleaban sus antiguos profesores cuando ellas y ellos cursaban estas etapas. La permanencia de viejos modelos educativos, basados en la enseñanza discursiva, no hace más que constatar la evidencia del fracaso educativo. El docente inhibe la participación de sus alumnos en el aula ocupando el tiempo escolar enseñando contenidos, la retención memorística de datos y 
proponiendo tareas rutinarias y aburridas. La resistencia al cambio, la acomodación o la desgana deja que el tiempo transcurra entre rutinas didácticas que ya eran antiguas cuando empezaron a desarrollarse. Críticas gratuitas como presuponer que las TIC en el aula pueden representar la trasferencia de una adicción (Adès y Lejoyeux, 2003) al espacio educativo, no pueden negar que el alfabetismo digital (Snyder, 2004) es una competencia básica necesaria en nuestro entorno cultural y que diseñar y poner en marcha procesos de enseñanza-aprendizaje en el aula, incentiva la vinculación entre la experiencia de vida de los estudiantes y los contenidos educativos. Además, proporciona un aprendizaje activo, donde el alumno no sólo escucha o toma notas, también estimula el pensamiento, lee, escribe, discute, reflexiona. Las herramientas digitales son elementos didácticos que proporcionan eficiencia. Tenemos que utilizarlas e incluso sacarlas de los bolsillos. No podemos seguir negando la posibilidad de que el alumnado utilice en clase los ordenadores portátiles (laptop) o los teléfono con conexión a internet (Smartphone) Son herramientas útiles que quizá en un primer momento puedan ser empleadas para actividades de ocio pero que, finalmente, se integrarán a las dinámicas de clase y a las tareas de aprendizaje. Los TIC constituyen recursos y medios en torno a materiales tecnológicos que vinculan la responsabilidad tanto del profesor con la enseñanza como del alumno con su propio aprendizaje (Dopico, 2010).

\section{DISCUSIÓN}

Cuando los/as alumnos/as van a realizar las prácticas externas a los centros educativos, se les asigna un tutor de la Facultad. Su tarea consiste en servir de referente para facilitar el acceso al centro y mantener activos los conocimientos adquiridos previamente, para que el alumno-docente en prácticas pueda cotejarlos en la práctica cotidiana con los aspectos conceptuales y metodológicos propios del desempeño profesional. El tutor de la Facultad actúa en coordinación con el tutor del Centro Educativo. Este será el profesor que pondrá al alumno-docente en contacto con el programa educativo, con las normas que regulan el funcionamiento del sistema y con el grupo-clase al que es asignado. Los niveles de cooperación que se establezcan entre ambos tutores, el de la Facultad y el de la Escuela, redundará en beneficio de la adecuada formación del docente en prácticas. No siempre ocurre. Los tutores de la 
Facultad no visitan a sus alumnos en prácticas las veces pactadas. Los tutores de los centros de Primaria y Secundaria no disponen del humor y el tiempo suficiente como para atender como sería deseable a jóvenes cargados de ilusión y conocimientos que preguntan mucho y quiere dar clase.

Una de las conclusiones inquietantes obtenidas al analizar los datos se relaciona directamente con el diseño de los programas formativos en la formación del profesorado. Entre el alumnado del Master (recordemos que se trata de licenciados universitarios), parece haber cierto desapego hacia los contenidos de aprendizaje. Esto se revela más notoriamente cuando regresan de hacer las prácticas externas en los centros, donde muchas de las creencias previas (creencia no es lo mismo que idea, por lo que las consideramos como razonamientos pedagógicos débiles), que poseen los estudiantes sobre la práctica de la enseñanza se reafirman tras su estancia en los colegios (Latorre, 2007). Si el conocimiento formal no les ayuda a solucionar los problemas que se encuentran, lo rechazan, retoman opiniones derivadas de su experiencia individual y argumentan sus decisiones basándose en razones personales.

En una lectura rápida de los datos presentados en esta investigación se podría formular una conclusión simplista: las Prácticas Externas son un componente esencial de la formación (González y Fuentes, 2011) y también son perjudiciales porque inducen a aceptar lo establecido (Zeichner, 1980). Ambos puntos de vista pueden ser apreciados en las respuestas obtenidas por los estudiantes. En parte es lógico que así sea pues no hay una visión unilateral de la educación ni una pauta única de entender la docencia. Aun así, es necesario tener claros al menos dos indicadores que contribuyen a aportar una buena formación práctica de quien se prepara para ser profesor/a durante las prácticas externas: de una parte, el hecho de conocer cuáles son las funciones y tareas que los profesores en prácticas pueden y deben realizar durante su estancia en los centros educativos; de otra, cuáles son las competencias profesionales (experiencias, habilidades, destrezas...) a adquirir en el intervalo que duran estas prácticas. No es posible, en ningún caso, abandonar la idea de la necesidad permanente de formación que tiene el profesorado. Tener vocación docente es importante, sentir atracción hacia la profesión estimula a los estudiantes a formarse con un alto nivel de motivación y esto hace que finalmente lleguen a convertirse en educadores los mejores (Manso y Ramírez, 2011). De hecho y desde la óptica del 
profesor competente, el profesor es el único estudiante en el aula. Mientras que los alumnos llegan, están un curso académico y luego siguen su camino, el buen docente contrae la responsabilidad permanente de seguir estudiando, de continuar adquiriendo competencias pedagógicas que le preparen para desarrollar su tarea con responsabilidad (Larrosa Martínez, 2010).

Es evidente que en la sociedad de la información, tanto las poblaciones analógicas (Ortoll, 2005), como los nativos digitales, han de estar alfabetizados. Esto es, ser competentes en la cultura letrada, en los textos electrónicos, en las tecnologías digitales, en los lenguajes audiovisuales, en el manejo de la información (Coll, 2005). Los alumnos-docentes en prácticas que acuden a los centros educativos a realizar las prácticas externas proceden de este mundo digitalizado. Los alumnos de Primaria y Secundaria que conformarán su grupo-clase de referencia y les esperan en las aulas, también comparten esa digitalización de su espacio vital. Pero el profesorado que trabaja en los centros aún es renuente a la utilización generalizada de las TIC en el espacio educativo. Y cuando lo hace, asocia esto con la simpleza de poner diapositivas en ppt para presentar su discurso. Esta es una de las censuras más significativas que hacen los alumnos-docentes en prácticas cuando vuelven a las aulas de las Facultades. El impacto que de hecho tienen las TIC en las teorías educativas, ha potenciado el interés por la construcción de unidades de conocimientos y el estudio de los procesos de enseñanza y aprendizaje escolar tanto en entornos presenciales como virtuales (Moreno Bailliére, 2002; Ruiz Lopes y Martínez López, 2010). Pero todavía no podemos hablar de su generalización en las aulas de Escuelas e Institutos. Y todo, a pesar de que los ambientes de aprendizaje que generan son altamente motivadores, de que también suponen una forma de organizar el proceso de enseñanza y que confieren responsabilidad al estudiante para participar activamente en la construcción de su propio aprendizaje 


\section{Bibliografía}

Adès, J. \& Lejoyeux, M. (2003). Las nuevas adicciones: Internet, sexo, juego, deporte, compras, trabajo, dinero. Barcelona: Kairós

Barberà, E. (2008). Calidad de la enseñanza 2.0. RED. [Documento en PDF]. Revista de Educación a Distancia, VII. Recuperado de http://www.um.es/ead/red/M7/elena.pdf

Bruner, J. S. (1996). The Culture of Education. Cambridge, MA: Harvard University Press.

Day, C. (2006). Pasión por enseñar. La identidad personal y profesional del docente y sus valores. Madrid: Narcea

Dopico, E. \& Garcia-Vázquez, E. (2010). Leaving the classroom: A didactic framework for education in environmental sciences. Cultural Studies of Science education, 7.

Gil Pascual, J. A. (2006). Estadística e informática SPSS en la investigación descriptiva e inferencial. Madrid: UNED

González Sanmamed, M. \& Fuentes Abeledo, E.J. (2011). El Practicum en el aprendizaje de la profesión docente. Revista de Educación, 354, 47-70. Recuperado de http://www.educacion.gob.es/dctm/revista-de-educacion/articulosre354/re35403.pdf?documentld=0901e72b811e1d2b

Hernández Sampieri, R. Fernández Collado, C. \& Baptista Lucio, P. (2000). Metodología de la Investigación. México: McGraw Hill

Larrosa Martínez, F. (2010). Vocación docente versus profesión docente en las organizaciones educativas. Revista Electrónica Interuniversitaria de Formación del Profesorado, 13 (4), 43-51. Recuperado de http://www.aufop.com/aufop/uploaded_files/articulos/1291992517.pdf 
Latorre Medina, M.J. \& Blanco Encomienda, F.J. (2011). El prácticum como espacio de aprendizaje profesional para docentes en formación. Revista de Docencia Universitaria, 9 (2), $35-54$.

Marcelo, C. (2009). Los comienzos en la docencia: un profesorado con buenos principios. Revista de Curriculum y Formación del Profesorado, 13 (1), 1-25. Recuperado de http://redalyc.uaemex.mx/pdf/567/56711733002.pdf

Martínez De Mlguel López, S. \& Escarbajal De Haro, A. (2011). La renovación de las metodologías docentes universitarias. Reflexiones desde el Grado de Educación Social. Revista Electrónica Interuniversitaria de Formación del Profesorado, 39 (144), 135-144. Recuperado de http://www.aufop.com/aufop/uploaded_files/revistas/132818819710.pdf

Martínez Olmo, F. (2002). El Cuestionario. Un instrumento para la investigación de las ciencias sociales. Barcelona: Laertes.

Moreno F. \& Bailliére, M. (2002). Diseño instructivo de la formación on-line. Aproximación metodológica a la elaboración de contenidos. Barcelona: Ariel.

Murillo, P. (2007). Nuevas formas de trabajar en la clase: metodologías activas y colaborativas. Madrid: M.E.C

Novella Cámara, A., Forés Miravalles, A., Rubio Serrano, L., Costa Cámara, S., Gil Pasamontes, E. \& Pérez Escoda, N. (2012). Innovar en, desde y para el practicum: supervisión y evaluación de la transferencia de competencias. Revista de Docencia Universitaria, 10 (1). 453-476.

Ortoll, E. (2005). Alfabetización y exclusión digital. Barcelona: Universitat Oberta de Catalunya

Pérez, C. (2005). Técnicas estadísticas con SPSS 12: aplicaciones al análisis de datos. Madrid: Pearson Alambra.

Pérez Gómez, A. (2012). Educarse en la era digital. Madrid: Morata

Puelles, M. (2009). Profesión y vocación docente: presente y futuro. Madrid: Biblioteca 
Nueva

Rodríguez, C.; Pozo, T. \& Gutiérrez, J. (2007). Fundamentos conceptuales y desarrollo práctico con SPSS de las principales pruebas de significación estadística en el ámbito educativo. Granada: Grupo Editorial Universitario.

Snyder, I. (2004). Alfabetismos digitales. Comunicación, innovación y educación en la era electrónica. Málaga: Aljibe.

Vaillant, D. \& Manso, J. (2012).Tendencias en la formación inicial docente. Cuadernos de Investigación Educativa, 3 (18). 11-30. Recuperado de http://www.ort.edu.uy/ie/pdf/cuad_18.pdf

Valle, J.M., \& Manso, J. (2011). La nueva formación inicial del profesorado de Educación Secundaria: modelo para la selección de buenos centros de prácticas. Revista de Educación, 354 (1), 263-290.

Visauta, B. (2007). Análisis estadístico con SPSS 14: estadística básica. Madrid: McGraw-Hill

Zeichner, K. (1980). Myths and realities: Field-based experiences in preservice teacher education. Journal of Teacher Education, 31(6), 45-55. 